\title{
Mechanical Characterization of Coatings Using Microbeam Bending and Digital Image Correlation Techniques
}

\author{
C. Eberl • D.S. Gianola • K.J. Hemker
}

Received: 19 May 2008 / Accepted: 22 September 2008

(C) Society for Experimental Mechanics 2008

\begin{abstract}
A new technique for characterizing end-supported microbeams of coating materials is presented. Microbeams are fabricated using micro-EDM machining to isolate the material under investigation from the underlying substrate. Three- and four-point bending is realized by a custom-built microspecimen testing system, and digital image correlation is employed to capture full-field strains and displacements in theses microbeams. These experiments provide the foundation for the use of finite element modeling and inverse methods to determine the mechanical properties (elastic moduli, strength, interfacial toughness) of the coatings. Here, the experimental details of the microbeam bending experiments are explained, discussed and illustrated through application to a multilayered metal/oxide/ ceramic thermal barrier coating system commonly used in aero-turbines.
\end{abstract}

Keywords Digital image correlation · Microbeam bending . Thermal barrier coatings - Aerospace materials .

Young's modulus $\cdot$ Fracture toughness

\author{
C. Eberl $(\bowtie)$ \\ Institut für Zuverlässigkeit von Bauteilen und Systemen, \\ Universität Karlsruhe, \\ Karlsruhe, Germany \\ e-mail: chris.eberl@kit.edu \\ C. Eberl $\cdot$ D.S. Gianola \\ Institut für Material Forschung II, Forschungszentrum Karlsruhe, \\ Karlsruhe, Germany
}

\section{K.J. Hemker}

Department of Mechanical Engineering,

The Johns Hopkins University,

Baltimore, USA

\section{Introduction}

The reliability of thermal barrier coatings (TBC) employed in the hottest sections of gas turbines for energy and aero applications has become increasingly more important as the application of these coatings have grown in recent years. TBCs provide greater durability and facilitate higher operating temperatures, which lead to increased fuel efficiency and reduced emissions. There is considerable interest in understanding failure mechanisms and developing models to predict TBC life $[1,2]$. These layered TBC systems consist of: a ceramic top coat, typically yttriumstabilized-zirconia (YSZ) deposited by either air plasma spraying (APS) or electron-beam physical vapor deposition (EB-PDV) [3, 4]; an intermetallic bond coat, e.g. $\mathrm{Ni}(\mathrm{Co})$ CrAlY or Pt modified NiAl; and the Ni-base superalloy substrate (SA). A thermally grown oxide (TGO) of dense alumina grows at the top coat/bond coat interface, reducing further oxidation and enhancing overall TBC lifetimes.

In service, the TBC is thermally cycled between room temperature and temperatures approaching $1,200^{\circ} \mathrm{C}$. Growth of the TGO and interdiffusion with the substrate often dramatically change the composition, microstructure and mechanical behavior of the bond coat $[5,6]$. The elevated temperatures also promote sintering of the YSZ in the top coat, which also causes dramatic changes in properties. EB-PVD coatings grow very columnar and have desired lateral compliance, but sintering leads to joining of columns, reduction in elastic anisotropy, the introduction of mud cracks and overall decreases in the performance of the top coat.

Models for life time predictions of TBCs rely on the availability of material properties, and experimental methodologies are needed for measuring and characterizing: coefficients of thermal expansion (CTE) as a function of 
temperature for all layers; Young's modulus and Poison's ratio for all layers as a function of temperature and geometric orientation; the yield and creep strength of the bond coat, TGO and top coat, as well as, bond coat/TGO and TGO/top coat interfacial toughness. This paper outlines a proposed microbeam bending test methodology for determining the elastic properties and strength of EB-PVD top coats, as well as the energy release rate associated with delamination of the bond coat/TGO interface. The "Background" section reviews previous approaches to measuring the properties of ceramic top coat materials, highlighting the difficulties of extracting properties from brittle, porous, multilayer materials. The "Materials and Specimens" section describes the microbeam geometry and fabrication route, and the subsequent sections describe the experimental setup and analysis procedure utilizing digital image correlation (DIC). Examples are presented to demonstrate the use of this method to determine the data needed to calculate elastic top coat properties and interfacial crack growth with inverse methods. The "Concluding Remarks" discuss the advantages of the testing methodology and propose possible improvements to the system.

\section{Background}

The implementation of bending experiments to extract elastic and plastic behavior from brittle or complex materials goes back to the beginning of the twentieth century as reviewed by Mayville and Finnie [7]. Mayville and Finnie use the analysis of Herbert [8] to measure the stress strain behavior of different materials by four-point bending and compare the results with conventional uniaxial tensile tests. It turns out that the difference is rather small and by using a bending experiment the materials could be tested up to much higher strains where in tension the samples fractured. Besides expressing experimental challenges during the test, Mayville and Finnie concluded that the test would be particularly useful to obtain material parameters for finite element (FE) calculations since even brittle metals like Beryllium could easily be characterized. Laws [9] points out that the higher failure strains measured in bending have to be taken with care as tensile loading typically cannot reproduce these values [10]. Another attempt to measure small amounts of plasticity was documented by Piggott [11] by wrapping sheet materials around rods with different diameters and measuring the resulting post-deformation radii of the sheet material after unloading. Piggott showed that this allows for the determination of plastic strains as small as $10^{-5}$. Brunet et al. [12] also used bending unbending tests to determine the plastic constitutive parameters needed for FE simulations of sheet metal deformation behavior. On the other hand, advanced strain measurement techniques, e.g. full-field DIC, allow for the use of complex sample geometries in order to extract elastic and plastic properties by inverse FE calculations [13] or other techniques like the virtual fields method presented by Grédiac and Pierron [14] from the experimentally determined full-field strain measurement.

The next logical step is to use the combination of DIC and bending techniques to measure complex material systems at small scales. DIC is a non-contact method, which allows measurements independent of the sample size and that is only limited by the resolution of the imaging system. And in combination with inverse FE calculations it is possible to extract elastic and plastic properties from bending experiments. In this paper we present the experimental details for measuring these values for a thermal barrier coating, as it combines complex material behavior on a small scale and furthermore satisfies the great need to provide reliable materials properties for these systems to predict life times of these systems.

\section{Material and Specimens}

The TBC evaluated in this study were provided by colleagues at Pratt \& Whitney and consisted of a $110 \mu \mathrm{m}$ $7 \%$ YSZ EB-PVD top coat, a $120 \mu \mathrm{m}$ thick low-pressure plasma sprayed (LPPS) NiCoCrAlY bond coat, and a Nibase superalloy substrate (PWA 1484). All coatings were characterized in the as-fabricated state (without thermal cycling) and a thin TGO $(t<1 \mu \mathrm{m})$ was observed to have formed between the bond coat and the top coat.

The specimen geometry was designed to isolate the ceramic top coat from the underlying superalloy. It was also important to test the materials in a typical configuration produced by industry to mimic the stress conditions seen in service; burner rig bars $(12.5 \mathrm{~mm}$ in diameter and roughly 120-150 mm long) were fabricated at Pratt \& Whitney and cross-sectioned to make specimens for the current study. Figure 1 illustrates the microbeam geometry and shows four specimens patterned into a prepared disk with a thickness of $\sim 550 \mu \mathrm{m}$. These microbeams were realized by carving out a wedge of the substrate under the coatings and leaving a doubly-clamped suspended beam, consisting of a ceramic top coat supported by a thin bond coat layer. The wedge provides an opening to insert a hook for loading using positioning stages and a custom built load frame.

A micro-electro-discharge machining process was utilized to fabricate the specimen, which allowed for precise control of the underlying bond coat layer thickness $(15<t<$ $60 \mu \mathrm{m})$. A typical as-fabricated specimen is shown in Fig. 2. This process has a minimal heat-affected zone and results in a beam geometry that follows the curvature of the bar sample. The top coat and bond coat layer both had 


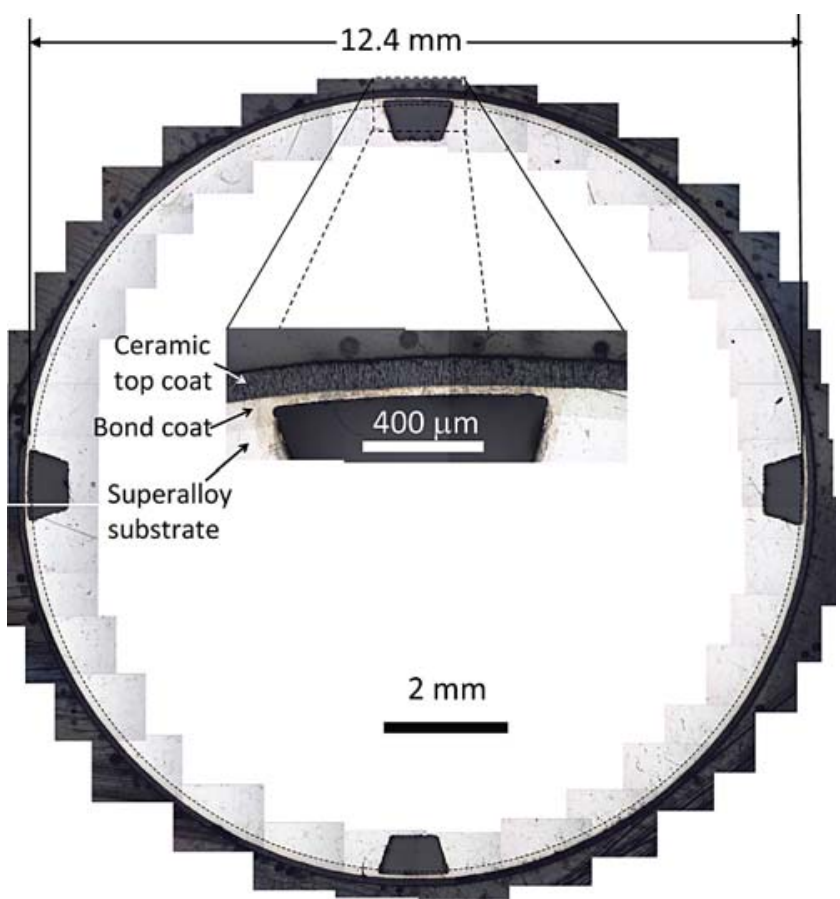

Fig. 1 Photo montage of multilayer disk specimen highlighting outer coatings. Four microEDM wedges are fabricated per disk specimen to create doubly-supported microbeams that primarily isolate ceramic top coat

sufficient natural material contrast for DIC when viewed with a high-resolution optical microscope and did not require any additional surface treatment.

\section{Loading System}

A typical macroscopic beam bending experiment requires the specimen, a loading system, and independent force and

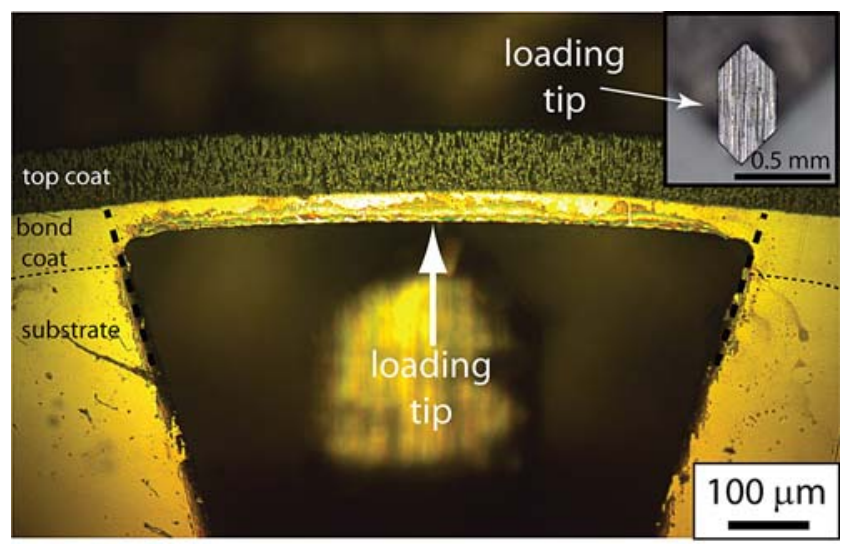

Fig. 2 Optical micrograph of three-point microbeam bending experiment. The loading tip is in place for testing, and is out-of-focus since the tip surface is higher than that of the specimen. The inset shows one design of the loading tip in focus measurement components. In conventional three- and fourpoint bending, wedge knife or roller pin geometries are used to apply point loads to the specimen, and either the orientation of the grips or the specimen can be modified to apply tensile or compressive stresses to desired regions. The multi-layered coatings studied here do not allow for such flexibility, although applying both tension and compression to the outer surface of the top coat is of great interest given the heterogeneous and anisotropic nature of the top coat microstructure.

In principal the load can be reversed by either: (1) pushing with a loading tip on the top coat surface to induce predominantly compression in the center span of the top coat, or (2) pulling from the bond coat side to induce tension in this region. The former approach is not ideal for the given example material system since the contact of the tip with the columnar top coat would create ill-defined stress concentrations and result in crushing of the top coat. For this reason, the pulling configuration was adopted for all experiments presented here. Furthermore, the fixed boundary conditions at the shoulders of the beams provide a gradient in the stress along the longitudinal axis when a point load is applied, transitioning from tension at the surface of the top coat in the center to compression in the shoulder regions (at the end constraint). The strain distributions of the microbeams under three- and four-point bending are shown in Fig. 3, as calculated by FE simulations of the multi-layers. The FEM simulations were carried out by the use of commercial software package (ANSYS). PLANE82 elements were used for the 2D simulation and the geometry was extracted from existing samples. For the as deposited bond coat a Young's modulus of $155 \mathrm{GPa}$ [15] and a Poisson ratio of 0.3 was used, for the top coat porous YSZ properties were estimated with a
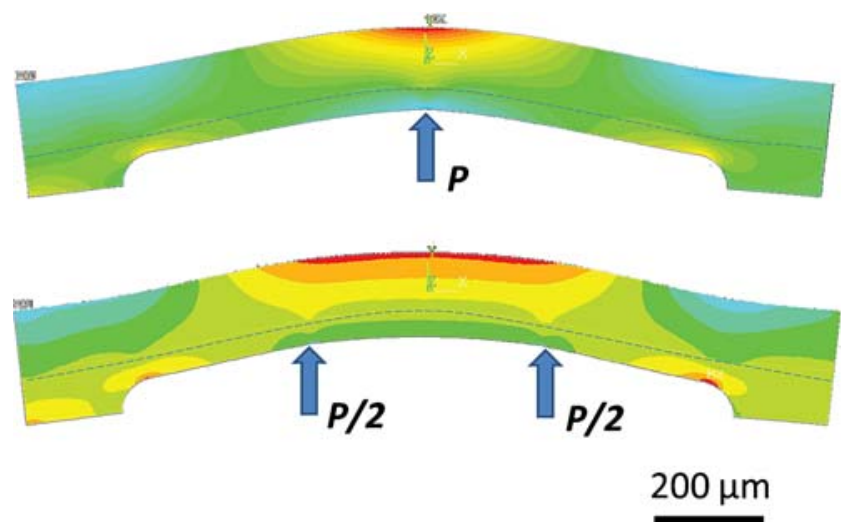

Fig. 3 (Color online) Finite element axial strain profiles for three- and four-point bending of a bilayer top coat/bond coat microbeam. Red colors represent tension $\left(4 \times 10^{-4}\right)$ and blue represent compression $\left(-4 \times 10^{-4}\right)$ for a load of some Ns. Note the transition from tension at the top coat surface in the center of the beam to compression in the shoulder region 
Young's modulus of $20 \mathrm{GPa}$ and a Poisson ratio of 0.3 . These values were chosen for initial simulations in order to get rough estimates of the strains and the needed forces for the experiments. These simple simulations cannot be used to analyze the elastic properties of the top coat or the energy release rate during interface crack propagation. A detailed description of such simulations are out of the scope of this paper but will be explained in upcoming publications. The features were exploited further for measurements of the asymmetric elastic behavior, and high-resolution DIC analyses were performed both in the center and shoulder regions of the microbeams.

Figure 4 shows an image and schematic of the testing system. The loading tip was fashioned as a "hook" that protrudes from a machined grip (Fig. 2). A single-point hook was used for three-point bending, and a double-point hook for four-point bending. The hook is attached to a positioning stack consisting of a single-axis piezoelectric actuator (New Focus 8302) for displacement-controlled testing, and a 5 -axis positioning stage (New Focus 8082) for precise alignment of the tip. The 5-axis stage allows for the tip to be brought up through the machined gap (Fig. 2) with the aid of a microscope without damaging the specimen, and also for the tip to be shifted laterally along the beam for fracture propagation experiments. A capacitance probe (Capacitec 410-SC) monitors the displacement of the system for real-time output. The other side of the load train consists of the specimen, which is mounted to a grip via a screw through a central hole, an air bearing for minimizing friction (Nelson Air, RAB1), and a $5 \mathrm{kgf}$ load cell (Entran, ELHS).

All specimen placement, alignment, and testing occurs under the observation of a high resolution Nikon microscope. The loading tip extends above the plane of the specimen when in its correct position, and the microscope has limited depth-of-focus at high magnification, so a series of images are used to position the tip appropriately between the shoulders of the specimen. Reflections and shadowing effects from the tip can preclude accurate strain measure- ment in the bond coat (closest to the tip) unless the tip is coated with black paint to minimize these effects.

A typical experiment is conducted as follows. The hook is positioned in the center of the beam as described above. The single-axis actuator is then driven slowly into the bond coat until a small level of force is recorded. Several approaches of the tip are made to allow for any tip "settling". The specimen is then loaded in $0.01 \mathrm{~N}$ increments, and after a short relaxation period, a series of nine digital images are captured. This experiment is conducted with the camera focused on the center of the beam, and then a second series of images taken with the focus on the shoulder.

\section{Displacement and Strain Measurements}

Non-contact strain measurements are necessary when measuring properties at the micro-scale and below since the specimens become very fragile. Image-based methods are ideal because of the relatively low cost associated with digital cameras and normal optical components, but also because they allow simultaneous measurement and observation of the experiments as they progress. Another requirement is a strain measurement approach with full-field capability, which allows one to capture both local events and global gradients. DIC satisfies these criteria and is relatively simple to implement.

\section{Digital Image Correlation}

DIC as a technique shows increasing impact as computers are getting faster, DIC code becomes available and optimized, and the advantages and capabilities are realized. Several authors have comprehensively reviewed the technique [16-18], therefore only a short introduction will be given here. DIC allows for non-contact strain measurement, which allows one to test on multiple scales (from nanometers to meters) and at various temperatures, pressures

Fig. 4 Image (a) and schematic (b) of system mechanical testing apparatus modified for microbeam bending experiments. The major components required for accurate testing are labeled

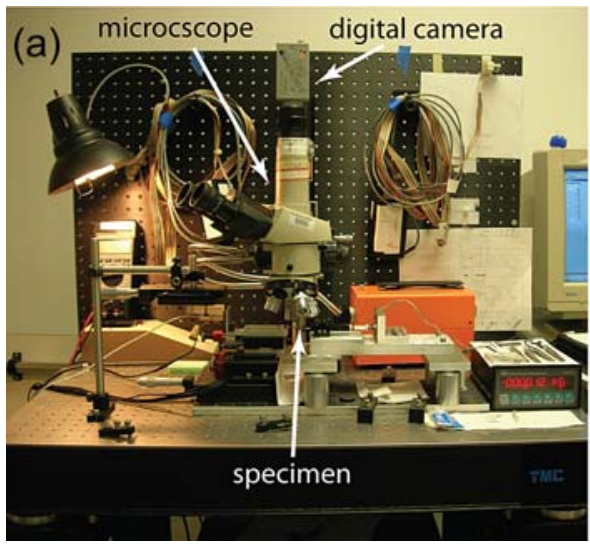

(b)

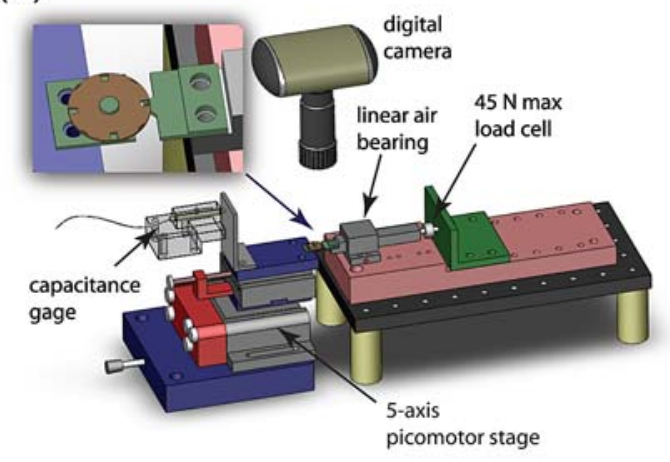


and atmospheres. The input images can be acquired with optical, electron, or atomic force microscopy, and the technique allows one to measure $2 \mathrm{D}$ and $3 \mathrm{D}$ strain fields in order to observe complicated test structures under multiaxial load.

In the study reported in this paper we have used DIC for micron-scale testing to measure heterogeneous structural material properties. There are some technological boundary conditions which should be noted at this point. DIC is based on the correlation of image subsets (from now on named markers), which define the maximum traceable displacement in the range of the marker. Subsequent shifts between consecutive images that are larger than the marker size cannot be tracked by this technique. Therefore, the image capture frequency should be chosen in a way that the subsequent displacement is smaller than this maximum value. Furthermore, the size of the tracked marker also increases the displacement resolution as a larger marker also means a larger data set for subset correlation. The markers used for this paper had sizes ranging between $20 \times$ 20 and $50 \times 50$ pixels $^{2}$ per marker, which were defined before the analysis in the DIC code. Rigid-body rotation in images cannot be detected here and lead to an experimental error. This has to be taken into account for rotations higher than $2^{\circ}$ to $3^{\circ}$ as image correlation typically uses the first image as a reference. Large deformations also pose a challenge since deformed subsets may be too large relative to their reference subset, which could lead to artificial stepwise displacements, as the same image subset is deformed more and more. One approach to avoid this problem is to correlate consecutive images, as opposed to always using the first image as the reference. This can be easily realized by changing the Matlab ${ }^{\circledR}$ code which was used in this paper, however, the microbeams studied here incurred small strains, and the effects described above were negligible.

The DIC analysis was conducted in a post process by utilizing a Matlab ${ }^{\circledR}$-based code developed at the Johns Hopkins University. This tool can be downloaded for free (http://www.mathworks.com/matlabcentral/fileexchange/ loadFile. .do? objectId=12413\&objectType=file///).

It should also be noted that the lighting conditions play a crucial role in terms of DIC accuracy. Changing light conditions during the experiment can lead to errors in the sample surface tracking and lead to false results. A good analysis requires a validity check by plotting the displaced markers on the associated images and tracking their synchronous movement. Spurious movements can be detected (either manually or automatically), and the corresponding markers can be removed from the analysis.

A low density marker mesh is shown in Fig. 5, where the blue dots represent markers distributed over the sample.
Fig. 5 Micrographs showing microbeam loading configuration and strain analysis. A raster grid (coarsened for clarity) for DIC is superimposed in (a) over both the top coat and bond coat materials. Regions for high-resolution analysis are shown: (b) center region where tensile strains develop at top surface of top coat, and (c) shoulder region where compressive strains develop at top surface of top coat
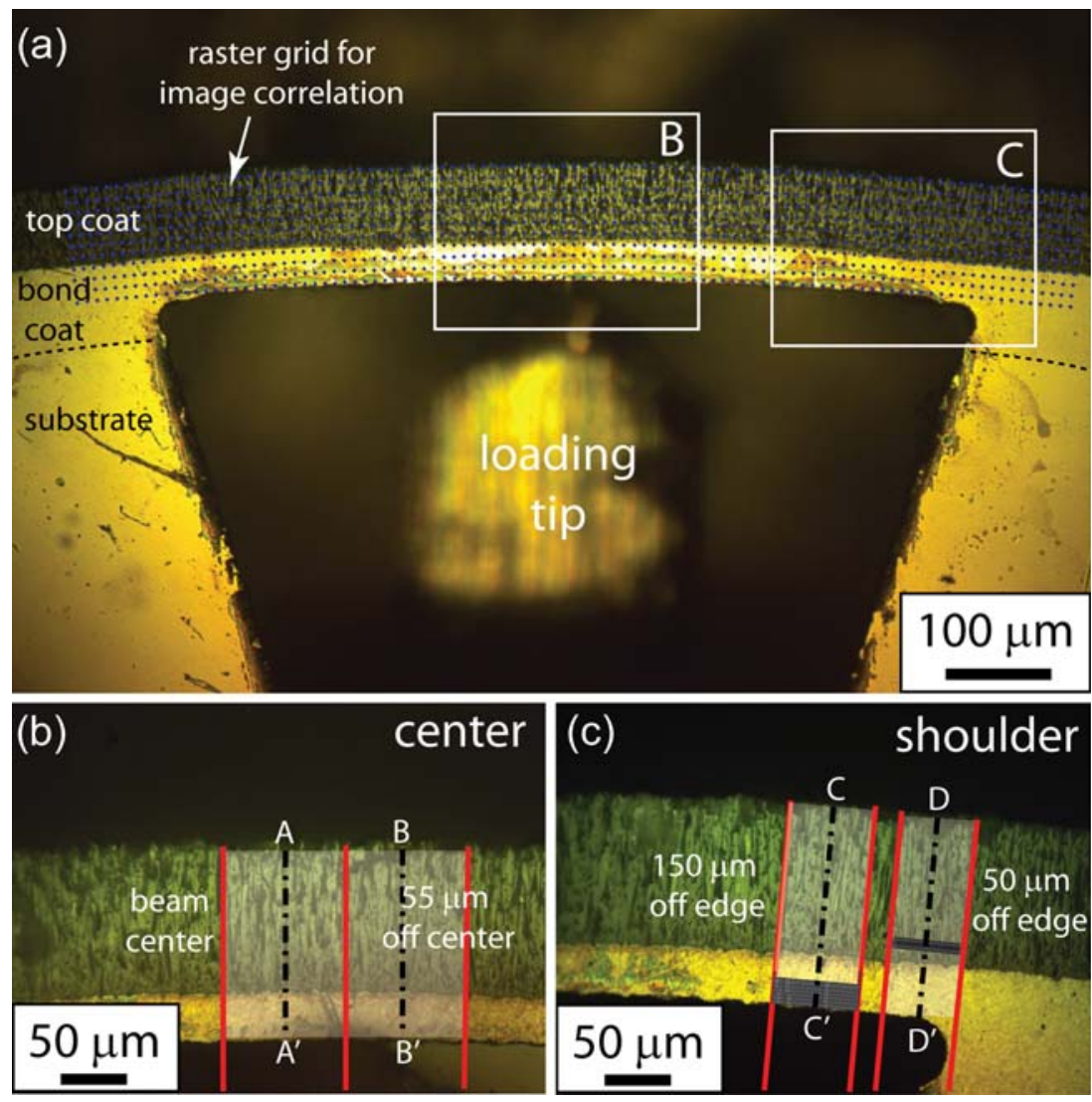
These markers represent the position of each image subset which will be tracked. The minimum density will be defined by the needed strain/displacement resolution, while the maximum density will be determined by the computational expense. In order to circumvent any smoothing effects, the density and size of markers should be chosen to avoid marker overlap.

For this study, a regular grid was generated with a marker spacing of 20 pixels in the horizontal and vertical direction of the image, leading to an overlap when using our standard marker size of $40 \times 40 \mathrm{pixel}^{2}$. For a typical analysis, roughly 10,000 markers were used per image. The DIC was then conducted on a multi-processor (2-4) machine. Before the detailed analysis, several approaches were employed to delete markers that did not track properly. The user of the software has to be careful at this step and ensure only experimental artifacts get deleted, otherwise useful information may get lost. After this step, the displacement noise was further reduced by averaging the marker positions for the multiple images captured during each load step.

\section{Optical Setup}

Great care has to be taken with the acquisition of the images as the required displacement resolution is in the sub-micron regime. Therefore a high-quality Nikon microscope (Fig. 3) was employed at its highest magnification of $\times 40$. Between the camera and the $\times 40$ objective, $a \times 0.7$ adapter was mounted to focus the image onto the CMOS chip of the digital camera (Pixelink, PL-782A). The pixel pitch size resulting from the interpolated CMOS Bayer pattern is $3.5 \mu \mathrm{m}$, resulting in approximately 8 pixels per micron for the given magnification. Although it could be argued that 8 pixels per micron is not sufficient for submicron resolution, as the wavelength and the quality of the optical system will never translate into a sharp image at this resolution, the displacement information is still present and the DIC still can track gradual changes on this scale independent of image sharpness. To reduce the influence of the load train on image quality, the load was applied in steps. To minimize the effect of image noise from the camera's CMOS chip and vibration from the air damped table $9 \times 8$ images were taken per load step. The software of the camera was used to average over eight images and the resulting nine images were processed by the digital image correlation. The resulting displacement field of these nine averaged images was then averaged again resulting in a mean displacement field for each load step. The load values were averaged corresponding to the procedure described above.

It is important to consider the typical displacement resolution obtained by this method. Figure 6 shows the axial displacement field as a function of position obtained from the correlation of consecutive images (averaged) in the unloaded state, which demonstrates the "noise floor" of the system. Good root-mean-square (RMS) displacement resolution over the entire field can be obtained only after utilizing routines for eliminating poorly correlated points from the analysis. Despite the fact that the top coat provides sufficient natural contrast for DIC, this is especially important when measuring strain in porous materials (as in the case of the top coat), since the pores do not provide any optical contrast for correlation. In our system, we have
Fig. 6 (Color online) Axial displacement field plotted in the unloaded state to demonstrate noise floor. Control points are projected onto $u_{x x}-y$ plane (shown in black) to show typical scatter

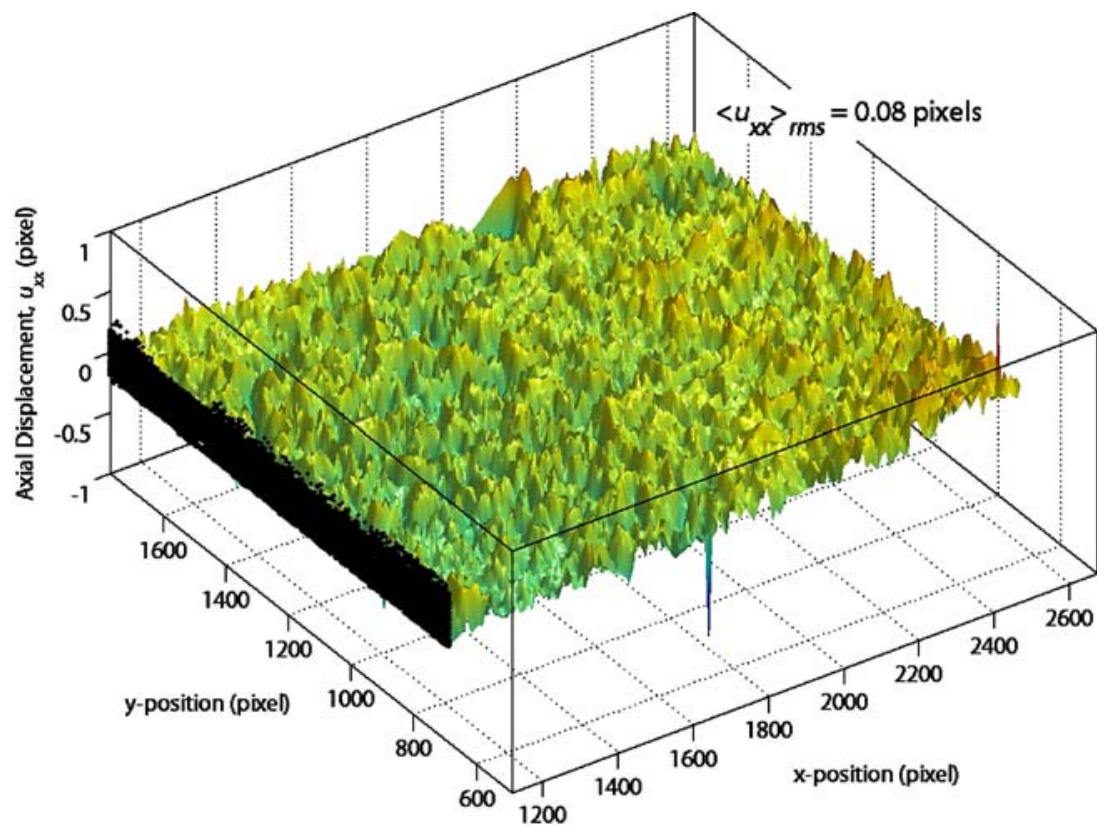


measured an RMS displacement of 0.08 pixels (corresponds to $\sim 10 \mathrm{~nm}$ displacement at this magnification) in the unloaded state over more than 15,000 markers. The aspects contributing most to the noise in these experiments include camera noise, mechanical vibrations, out-of-plane displacements, distortions from the optical system, and changing lighting conditions. The resolution measured during our experiments enables the determination of small strains incurred in elastic tests and small crack openings during fracture propagation events.

\section{Experimental Procedure}

Tensile Elastic Properties of the Top Coat

To measure the tensile properties of the top coat, three-point and four-point bending modes can be used to induce tensile strains in the top coat layer at the beam center (see Fig. 4). After conditioning the displacement data (e.g. removing poorly tracked markers, averaging over the image series per load increment), the actual analysis can be conducted by selecting the region of interest. These sites were selected by picking regions that display prominent strain distributions in the FE calculations (Fig. 3). Representative regions are shown in Fig. 4(b) for the beam center. Two regions were selected, one at the geometric center along the path $\mathrm{A}-\mathrm{A}^{\prime}$ and a second along the path $\mathrm{B}-\mathrm{B}^{\prime}$ which is $55 \mu \mathrm{m}$ offcenter. After selecting a path and the surrounding markers from the displacement data, the strain was analyzed for a defined number of layers from the top coat surface to the bond coat surface for each load step (see Fig. 12 for the strain normalized by the load versus distance from the top coat surface).

\section{Top Coat Tensile Strength}

Several load-unload cycles to increasing maximum loads were carried out for each beam in three-point bending mode. Eventually a vertical crack was nucleated and observed to have run from the surface of the top coat to the top coat/bond coat interface formed. The actual crack propagation through the top coat was not resolved as the crack growth is too rapid and because image acquisition was started after a new load step was reached to minimize vibration. The spanwise position of these vertical cracks was observed to occur near to, but not exactly at the point of maximum tensile stress, owing to microstructural heterogeneities in the top coat. The axial strain of the last load step was recorded and together with the elastic properties of the top coat, used to estimate the top coat strength. Due to the high strain gradient induced by the three-point bending mode, this strength can be expected to be higher than for a bulk sample as the probed volume is very small. Assuming that the top coat tensile strength is Weibull distributed, a higher sample volume will lead to a lower mean tensile strength.

\section{Compressive Elastic Properties of the Top Coat}

The vertical top coat crack significantly reduces the stiffness of the whole system since the top coat is slightly debonded from the bond coat. This feature was exploited when measuring the compressive elastic properties of the top coat; higher strains were achieved in the shoulder sections because of this reduced stiffness. During shoulder experiments, two paths $\mathrm{C}-\mathrm{C}^{\prime}$ and $\mathrm{D}-\mathrm{D}^{\prime}$ were defined [Fig. 5(c)]. These sites were selected based on FE calculations as they predict prominent strain distributions from the top coat surface to the top coat/bond coat interface. Further analysis steps closely follow the description of the tensile elastic properties of the top coat.

\section{Top Coat/Bond Coat Interface Fracture Toughness} Measurement

The interfacial fracture toughness was determined by tracking the propagation of the crack along the interface during off-center loading conditions in three-point bending mode. By intentionally loading the beam in an off-center position, the vertical top coat crack can be driven into and along the TGO/bond coat interface. For each test the tip was moved to a new position, away from the perpendicular top coat crack, and then a stepwise loading applied until the crack propagated along the top coat/bond coat interface in the direction of the new load tip position top coat bond coat. Figure 7 shows an off-center load that has driven the interfacial crack (in the direction of the loading tip) from

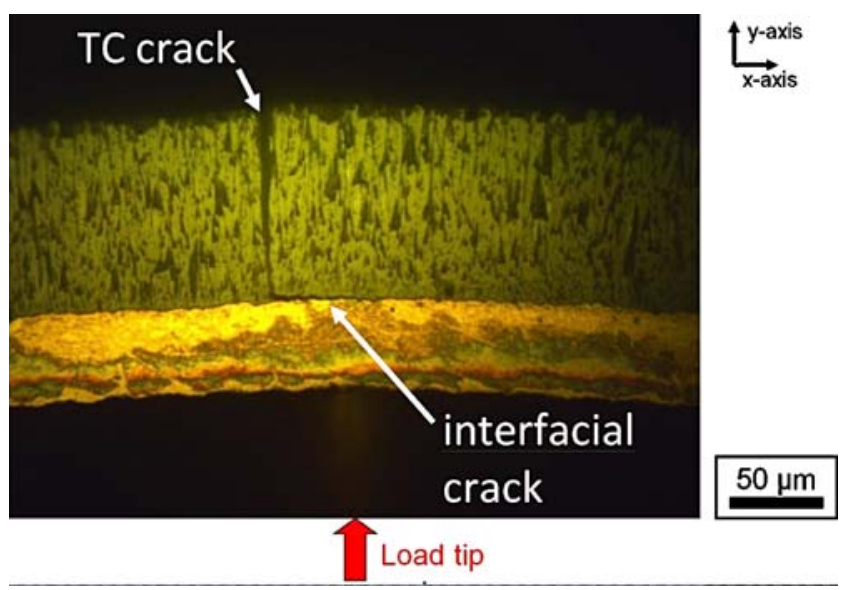

Fig. 7 Optical micrograph shows the off center position of the load tip during three-point bending crack propagation experiments. Here, both top coat and interfacial cracks have formed and have noticeable openings 
the vertical top coat crack. These steps can be repeated on both sides until the forces to drive the crack become too high as the load tip approaches the fixed shoulder of the beam.

The same test can be conducted in four-point bending mode after inducing interface cracks on both sides of the perpendicular top coat crack in three-point bending mode. This allows for a symmetric stress-strain field about the beam center, which can readily be estimated by analytical models. This circumvents the difficulties associated with iterative (inverse) numerical approaches to property extraction. The four-point bending configuration is shown in Fig. 8.

The displacement fields gained from the DIC can then be used to quantitatively characterize the crack propagation along the TGO/bond coat interface as a function of the applied load by the use of a Matlab ${ }^{\circledR}$ script. Since the displacement at the crack tip is quite small and the images only highlight the crack tip at one surface (as opposed to the through-thickness crack front), the collective structural deformation of the beam was used to determine the crack tip. This means that instead of simply tracking the surface crack opening displacement, the full displacement field along the crack was used to measure the displacement of the bond coat relative to the top coat. To increase the resolution of the crack opening displacement even further the cumulative crack opening displacement was used (see Fig. 13).

The typical testing and analysis procedure for these microbeam bending experiments is illustrated in Fig. 9. One side of the flow chart represents the procedure for measuring the elastic response of the coating system, while

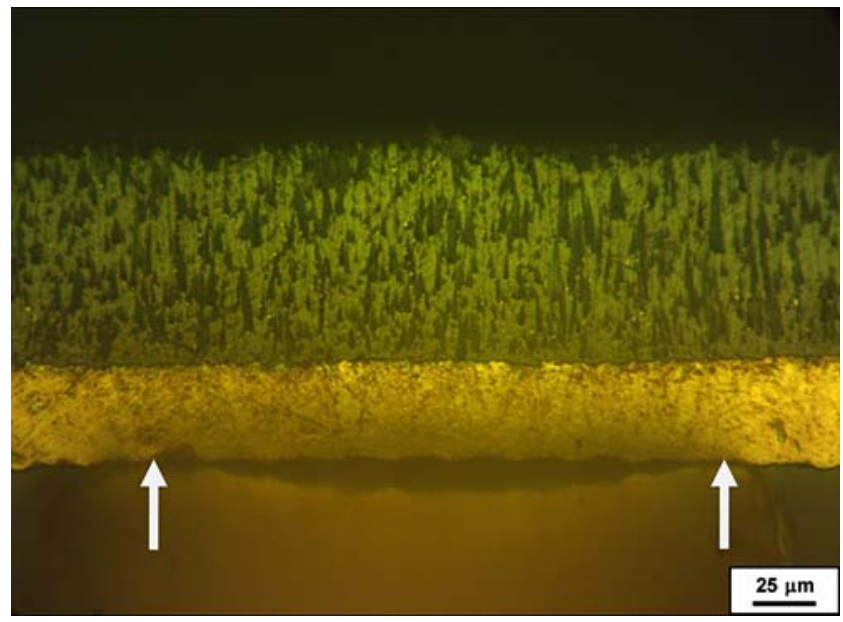

Fig. 8 Four-point bending configuration. The two-end bending tip cannot actually be seen, instead the bond coat surface of the sample is visible twice, once the real one (upper one) and a second time mirrored by the tip surface (lower one). The actual tip is out of focus and not visible

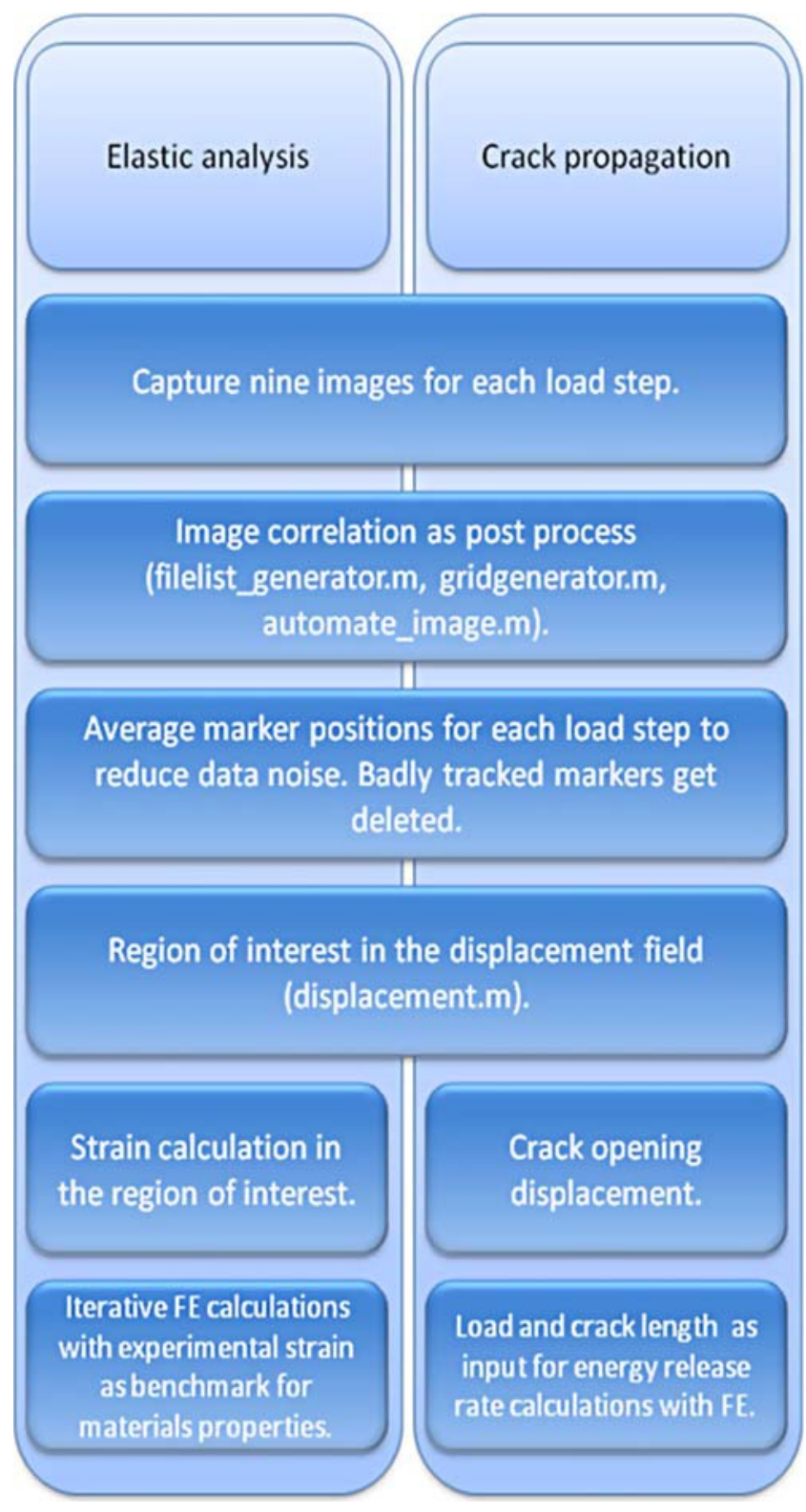

Fig. 9 Flow chart of analysis procedure (one side elastic, other side fracture). The names of the Matlab ${ }^{\circledR}$ scripts (http://www.mathworks. $\mathrm{com} / \mathrm{matlabcentral} /$ fileexchange/loadFile.do? objectId=12413\&objectType=file///) used for each step of the procedure are listed in parentheses

the other demonstrates the steps necessary to extract the fracture behavior of the top coat/bond coat interface.

\section{Results}

Elastic Deformation Measurements

Representative axial $\left(\varepsilon_{x x}\right)$ strain maps of the microbeam center during three-point bending at four different loads are plotted in Fig. 10. Tensile strains were incurred at the top 
Fig. 10 Image sequence showing micrographs with superimposed center strain maps for varying levels of loading: $P=$ (a) $0.9 \mathrm{~N}$, (b) $1.5 \mathrm{~N}$, (c) $2.4 \mathrm{~N}$, and (d) $3.4 \mathrm{~N}$. (e) Axial strain at the top coat crack position as a function of load, which clearly shows the crack nucleation event. Values beyond the cracking event do not represent real material strain. Behavior before crack nucleation is elastic as demonstrated by load/unload cycles performed in this regime
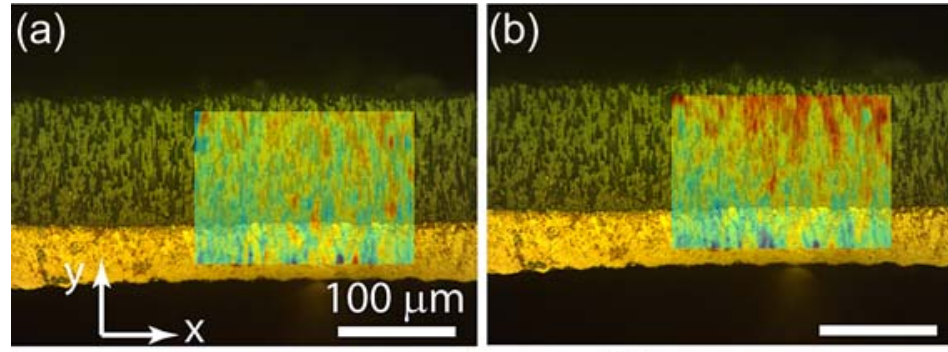

$\varepsilon_{\mathrm{XX}}$ $\times 10^{-3}$
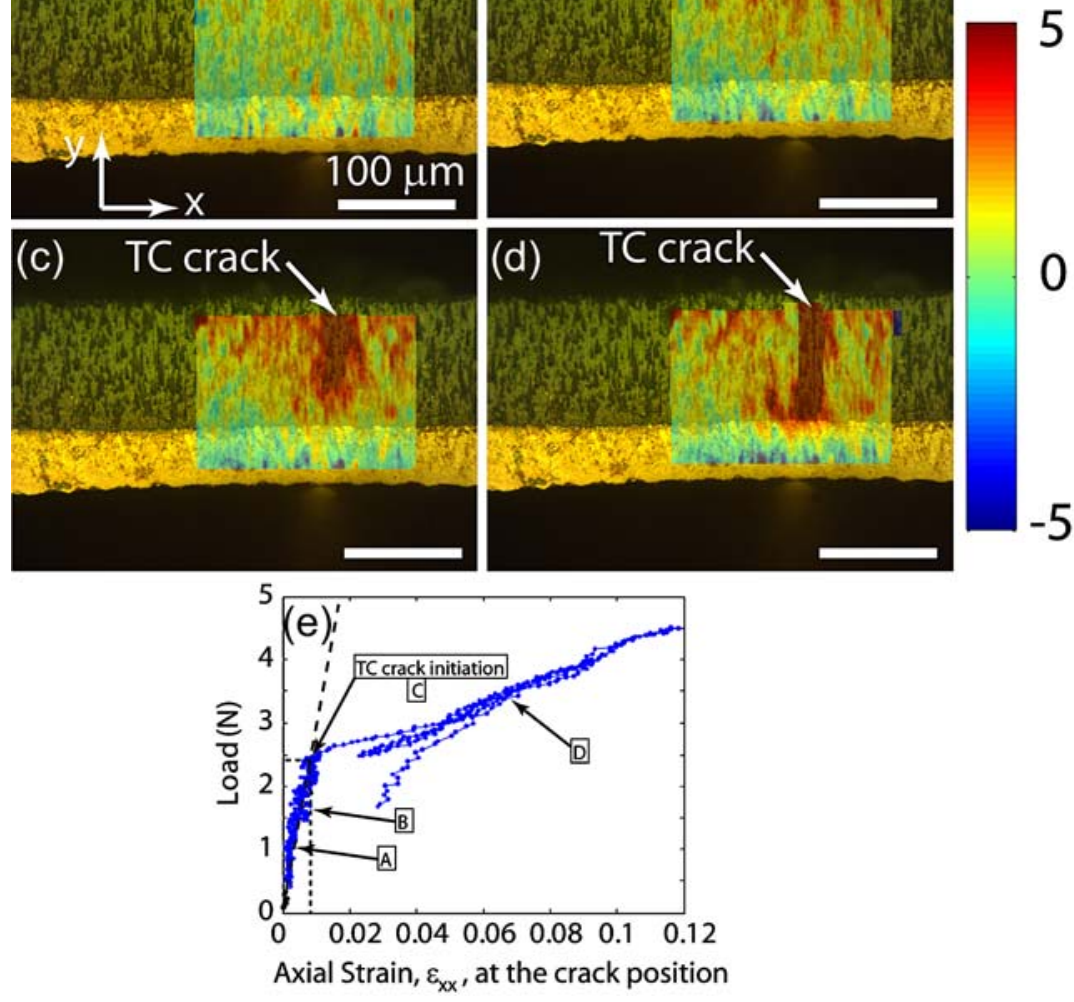

toughness of the ductile bond coat is sufficiently high to arrest the crack. Once the top coat vertical crack was nucleated, then the majority of the load was carried by the bond coat, as evidenced by the drop of the surface tensile strains (away from the top coat crack) in the top coat until the load was further increased. Furthermore, the maps in Fig. 10 show that the strain is heterogeneous at a local scale and appears to be striated in the transverse $(y)$ direction,
Fig. 11 Image sequence showing micrographs with superimposed shoulder strain maps for varying levels of loading: $P=$ (a) $1.0 \mathrm{~N}$, (b) $1.9 \mathrm{~N}$, (c) $2.9 \mathrm{~N}$, and (d) $3.4 \mathrm{~N}$
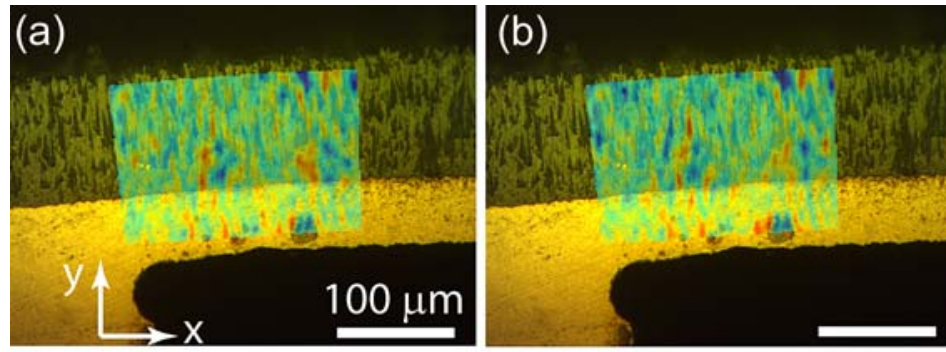

$\varepsilon_{\mathrm{XX}}$
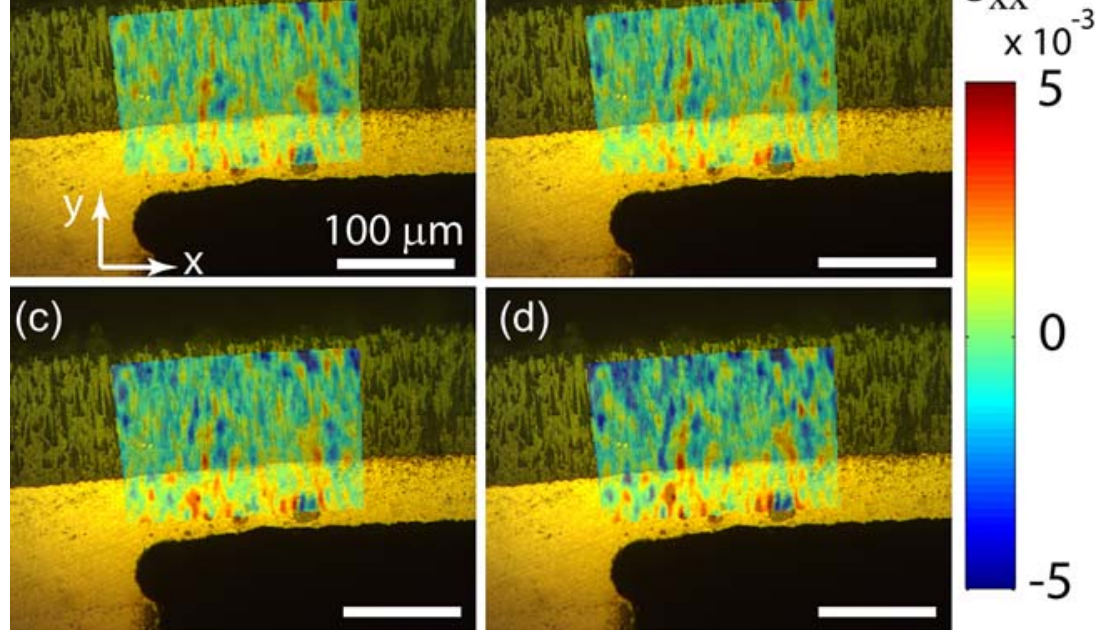


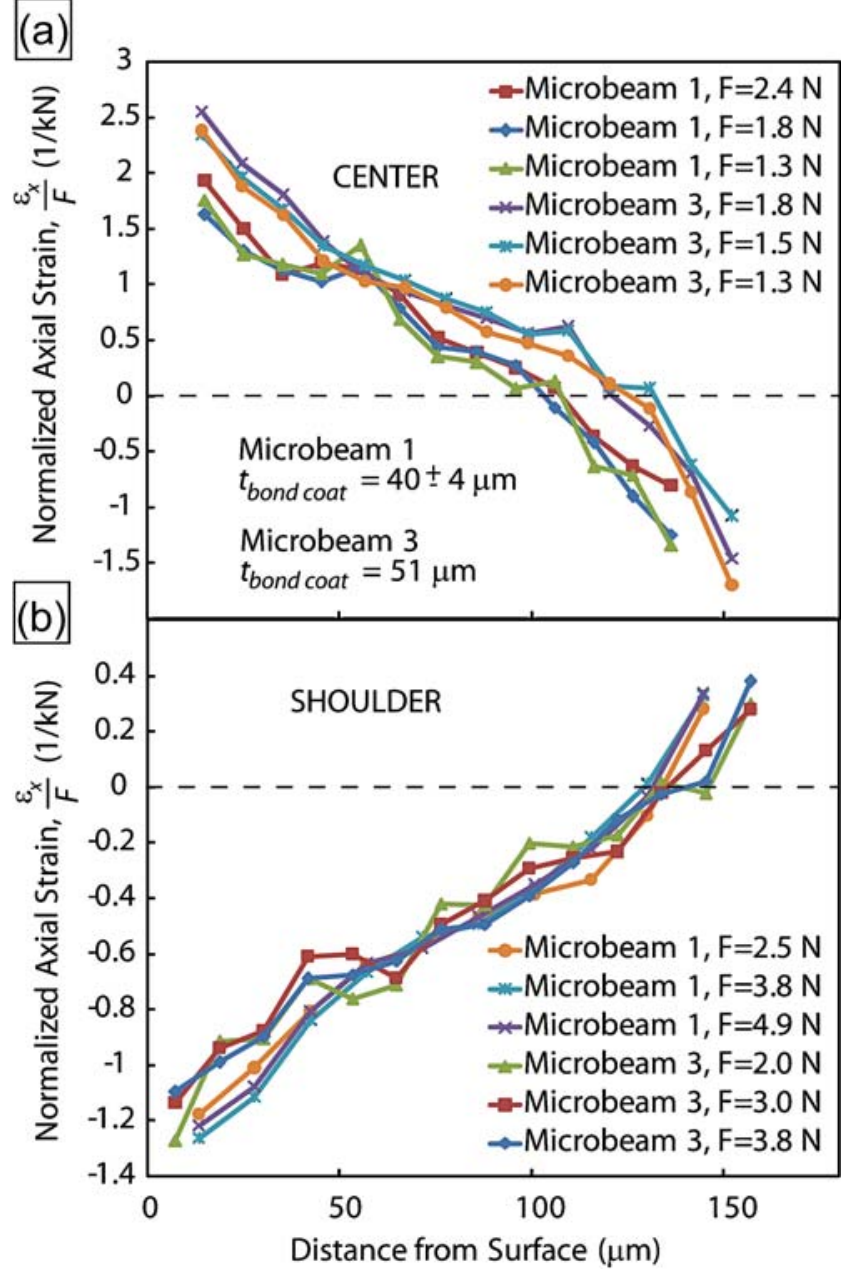

Fig. 12 Plot showing repeatability of measurements of elastic behavior in both the (a) tensile (center), and (b) compressive (shoulder) region where strains are small. The strain is normalized to the applied load to be able to compare the strain distribution for different loads. The dashed line shows the neutral axis corresponding to the columnar top coat microstructure. These observations, along with a plot of the load as a function of top coat surface strain [Fig. 10(e)], allowed for easy discernment of the top coat cracking event. All deformation prior to these events in virgin specimens was considered to be elastic, which is corroborated by elastic unloads/reloads performed [Fig. 10(e)] in this regime that follows the same loading path.

Compressive strains were observed in the top coat in the shoulder regions (at the ends of the microbeam) during the microbending experiments, and they were measured as shown in Fig. 11. Even though the strains in this region were very small $(<-0.5 \%)$, the image analysis is still able to discriminate the transverse strain gradient from compression at the top coat surface to tension in the bond coat. As expected, no cracking was observed in the shoulder regions since the top coat should be very damage resistant in compression.

Plotting the axial strain values in both the center and the shoulder region as a function of transverse position (distance from the top coat surface, where $x=0$ is the surface) gives the required input to extract the elastic properties of the top coat in tension and compression. Figure 12 shows the axial strain normalized by the applied load for two separate microbeam specimens. The data for each specimen collapses on the same curve for all loads suggesting that the elastic modulus of the top coat is not strain-dependent. The curves for the center specimens [Fig. 12(a)] have approximately the same slope but are offset because they contain bond coats of different thickness. By contrast the curves for the shoulder specimens [Fig. 12(b)] are not offset because the top coat is stiffer in compression and the majority of the load is carried in the top coat. The scatter in the curves in Fig. 12 is greater
Fig. 13 Image sequence showing micrographs with superimposed axial displacement maps for varying levels of loading. The load was (a) $0.28 \mathrm{~N}$, (b) $0.97 \mathrm{~N},(\mathbf{c}) 1.64 \mathrm{~N}$, (d) $2.23 \mathrm{~N}$
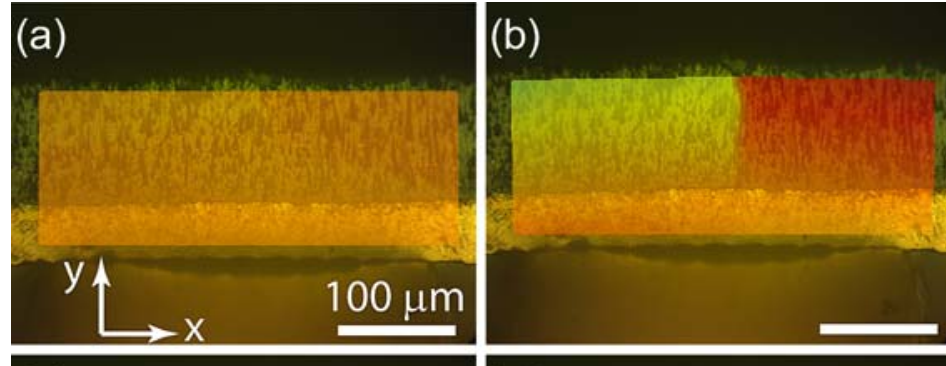
$u_{\mathrm{XX}}$ (pixels)
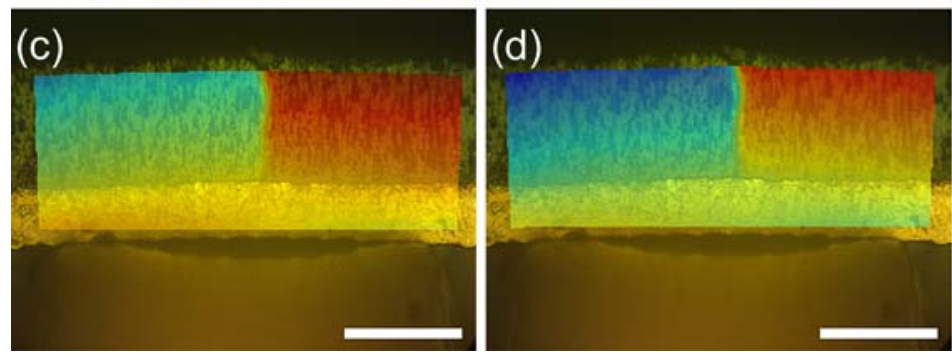
Fig. 14 Image sequence showing micrographs with superimposed center strain maps for varying levels of loading. Cracked regions show artificial apparent levels of strain that are beyond the color range. The load was (a) $0.28 \mathrm{~N}$, (b) $0.97 \mathrm{~N}$, (c) $1.64 \mathrm{~N}$, (d) $2.23 \mathrm{~N}$. (e) Magnification of the strain field shows in homogeneous deformation in the ceramic top coat at a load of $2.23 \mathrm{~N}$ on the structural level. (f) The strain analysis shows that the crack in the top coat has been driven to the interface were it extends along
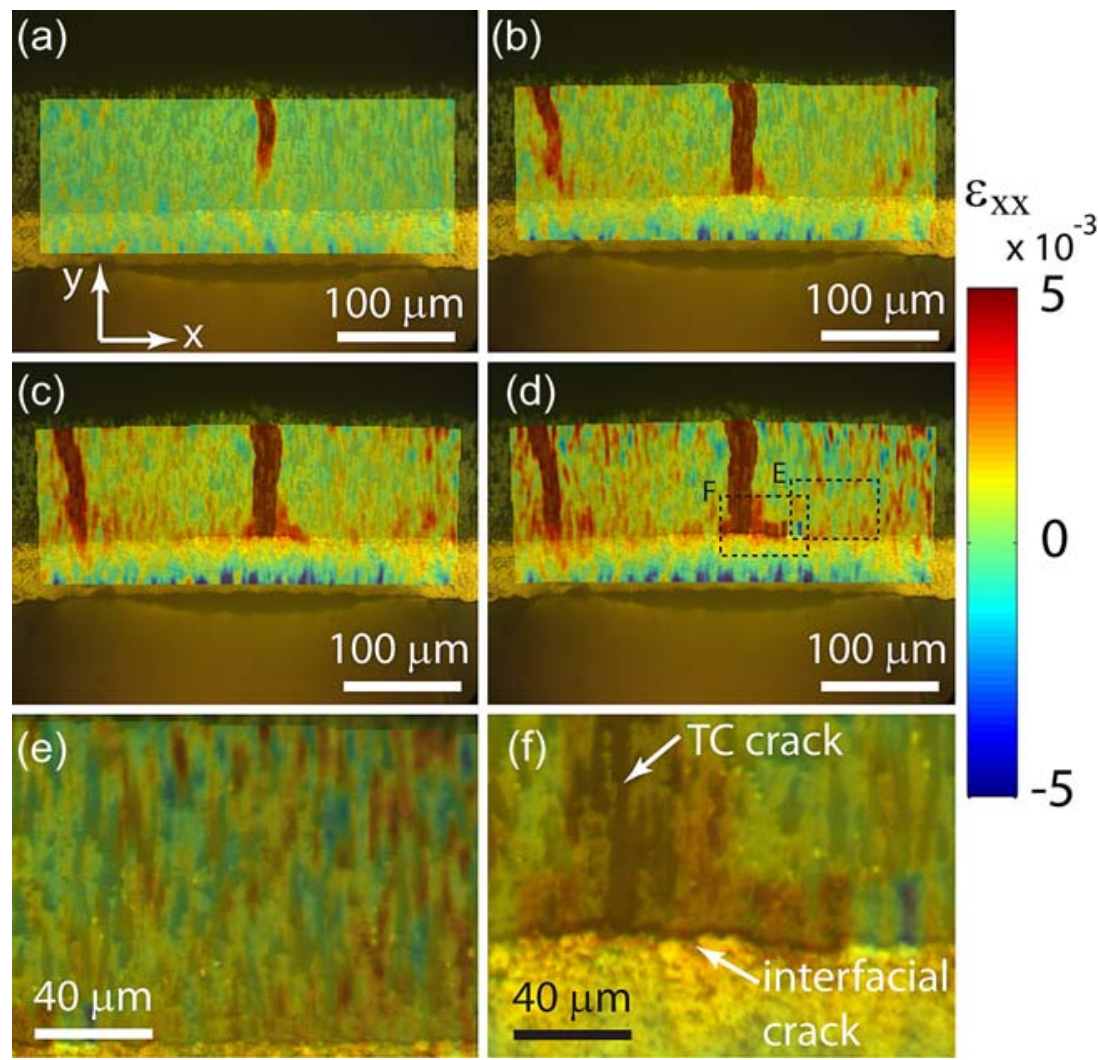

for the center data than the shoulder data. This may also be due to the difference between tensile and compressive loading. Inhomogeneities would be magnified more during tensile loading if the top coat was stiffer in compression. For a free-standing bilayer the Young's moduli and thickness of the individual layers are related to the position of the neutral axis as described in equation (1) [19]:

$\frac{h_{0}}{h_{\mathrm{BC}}}=\frac{1+\frac{2 \cdot E_{\mathrm{TBC}} \cdot h_{\mathrm{TBC}}}{E_{\mathrm{BC}} \cdot h_{\mathrm{BC}}}+\frac{E_{\mathrm{TBC}} \cdot h_{\mathrm{TBC}}^{2}}{E_{\mathrm{BC}} \cdot h_{\mathrm{BC}}^{2}}}{2 \cdot\left(1+\frac{E_{\mathrm{TBC}} \cdot h_{\mathrm{TBC}}}{E_{\mathrm{BC}} \cdot h_{\mathrm{BC}}}\right)}$

where $h_{\mathrm{BC}}$ is the position of the neutral axis, $h_{\mathrm{BC}}$ and $E_{\mathrm{BC}}$ are the thickness and the Young's modulus of the bond coat and $h_{\mathrm{TBC}}$ and $E_{\mathrm{TBC}}$ are the thickness and the Young's modulus of the ceramic top coat. Equation (1) provides a crude yet reasonable estimate of the Young's modulus of $E_{\mathrm{TBC}}=20 \pm$ $10 \mathrm{GPa}$, assuming isotropic elasticity $\left(E_{\mathrm{BC}}=155 \mathrm{GPa}[15]\right)$, small tensile elastic strains and homogeneous loading, the geometry of the specimens $\left(h_{\mathrm{BC}} \sim 50 \mu \mathrm{m}, h_{\mathrm{TBC}} \sim 110 \mu \mathrm{m}\right)$ and the neutral axes indicated in Fig. 12(a).

\section{Crack Propagation Measurements}

The details surrounding the initiation and propagation of interfacial cracks between the top coat and the bond coat are clearly elucidated by DIC analysis. Figure 13 shows the divergence of axial displacement after a top coat crack has been introduced. Observations such as this allow for easy determination of the position of a crack in the specimen. Figure 14 shows the evolution of the axial strain during crack propagation events, which provide sufficient resolution to reveal local strain information near an advancing crack. In Fig. 14, multiple cracks have initiated during fourpoint bending, although the primary crack is always near the center of the beam.

The interfacial crack propagation between the top coat/ TGO and bond coat is imaged as a function of loading in Fig. 15; however, a quantitative analysis of the fine crack propagation events requires DIC. The crack advancement events can be tracked by plotting the cumulative crack opening (crack opening displacement accumulated over load steps, Fig. 15). Furthermore, calculating the gradient of the cumulative displacement (the rotation across the interface) gives an indication of the "pivoting" caused by the crack. The red bars in the image indicate rotations on the order of $1^{\circ}$ to $10^{\circ}$. A large divergence of the bars (strongly opposing values) signifies a pivot point. This can be used to distinguish between the opening of a pre-cracked interface and the propagation of a crack leading to newly created surface. Using this approach, the pivoting of the top coat with respect to the bond coat allows us to track the relative displacement of the entire structure as opposed to simply the local opening. The subtleties of the crack 

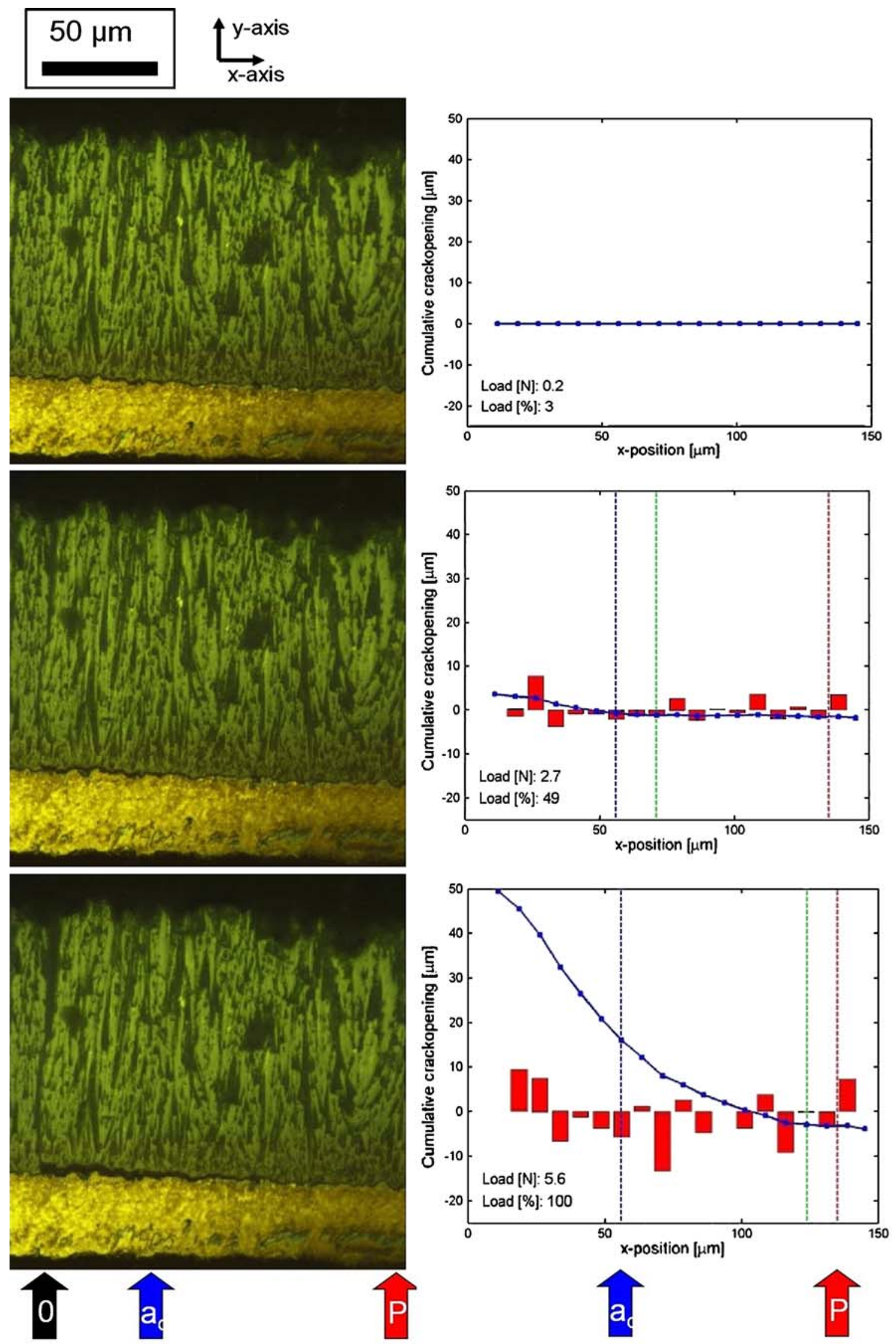
Fig. 15 (Color online) Image sequence showing interfacial crack propagation and cumulative crack opening displacement (blue line) and the differential displacement (red bars) as a function of position during a three-point bending experiment. The plot is shown for three loading steps at loads of $0.2,2.7$, and $5.6 \mathrm{~N}$

propagation events can be analyzed with high sensitivity using these metrics.

\section{Concluding Remarks}

Digital image correlation proves again to be an enabling technique which allowed us to investigate mechanical properties of complex materials and structures. As e.g. in thermal barrier coatings, anisotropic and inhomogeneous material properties can be characterized. Furthermore, digital image correlation is independent of scale and imaging technique and can be easily applied to small scale testing as shown in this paper in form of microbeam bending experiments. As increasingly complex materials and structures are analyzed, the interpretation becomes challenging and inverse FEM simulations with full field strain input from experiments have to be utilized to extract local materials properties. The results gained from this process are worth the effort as material properties in material systems can be evaluated without the need to separate the materials from each other. Due to these advantages, the micro beam bending technique presented here will be adopted to provide properties for other coatings and small scale systems in the future to enhance scientific and technical insights.

Acknowledgements This work was funded by AFOSR under the MEANS-2 Program (Grant No. FA9550-05-1-0173). The authors would like to thank A.G. Evans and J.W. Hutchinson for creative and enlightening discussions that allowed this technique to come to fruition. We also thank M. Maloney and D. Litton of Pratt and Whitney (USA) for providing the specimens, S. Faulhaber for preparing the disk specimens, J. Mraz (Smaltec) for fabricating the microbeam specimens, and W.N. Sharpe, Jr. and J. Sharon for technical support. C.E. would like to acknowledge partial financial support from the Max-Planck Society. D.S.G. acknowledges support from an Alexander von Humboldt Postdoctoral Fellowship.

\section{References}

1. Evans AG, Mumm DR, Hutchinson JW, Meier GH, Pettit FS (2001) Mechanisms controlling the durability of thermal barrier coatings. Prog Mater Sci 465:505-553
2. Evans AG, Hutchinson JW (2007) The mechanics of coating delamination in thermal gradients. Surf Coat Technol 201:79057916, doi:10.1016/j.surfcoat.2007.03.029

3. Levi CG (2004) Emerging materials and processes for thermal barrier systems. Curr Opin Solid State Mater Sci 81:77-91

4. Padture NP, Gell M, Jordan EH (2002) Thermal barrier coatings for gas-turbine engine applications. Science 296:280-284, doi:10.1126/science.1068609

5. Pan D, Chen MW, Wright PK, Hemker KJ (2003) Evolution of a diffusion aluminide bond coat for thermal barrier coatings during thermal cycling. Acta Mater 51:2205-2217, doi:10.1016/S13596454(03)00014-4

6. Mendis BG, Tryon B, Pollock TM, Hemker KJ (2006) Microstructural observations of as-prepared and thermal cycled NiCoCrAlY bond coats. Surf Coat Technol 201:3918-3925, doi:10.1016/j.surfcoat.2006.07.249

7. Mayville RA, Finnie I (1982) Uniaxial stress-strain curves from a bending test. Exp Mech 226:197-201, doi:10.1007/BF02326357

8. Herbert H (1910) Über den Zusammenhang der Biegungselastizität des Gusseisens mit seiner Zug- and Druckelastizität (On the connection between bending deformation and tension and compression deformation for cast iron), Mitt. und Forschungsarb. Veb. deut. Ing. 89, 39-81

9. Laws V (1981) Derivation of the tensile stress-strain curve from bending data. J Mater Sci 6:1299-1304

10. Allen HG (1971) Stiffness and strength of two glass-fiber reinforced cement laminates. J Comp Mater 5:194, doi:10.1177/ 002199837100500205

11. Piggott MR (1964) A method of determining plastic deformation in near-brittle materials. Br J Appl Phys 15:851-855, doi:10.1088/ 0508-3443/15/7/310

12. Brunet M, Morestin F, Godereaux S (2001) Nonlinear kinematic hardening identification for anisotropic sheet metals with bending-unbending tests. J Eng Mater Technol 123:378-383, doi:10.1115/1.1394202

13. Meuwissen MHH, Oomens CWJ, Baaijens FPT, Petterson R, Janssen JD (1998) Determination of the elasto-plastic properties of aluminium using a mixed numerical-experimental method. J Mater Process Technol 75:204-211, doi:10.1016/S0924-0136(97) 00366-X

14. Grédiac M, Pierron F (2006) Applying the virtual fields method to the identification of elasto-plastic constitutive parameters. Int $\mathrm{J}$ Plast 22:602-627, doi:10.1016/j.ijplas.2005.04.007

15. Hemker KJ, Mendis BG, Eberl C (2008) Characterizing the microstructure and mechanical behavior of a two-phase NiCoCrAlY bond coat for thermal barrier systems. Materials Science and Engineering A 483-484:727-730, doi:10.1016/j.msea.2006. 12.169

16. Peters WH, Ranson WF (1982) Digital imaging techniques in experimental stress analysis. Opt Eng 21:427

17. Chu TC, Ranson WF, Sutton MA (1985) Applications of digitalimage-correlation techniques to experimental mechanics. Exp Mech 25:232, doi:10.1007/BF02325092

18. Bruck HA, McNeill SR, Sutton MA, Peters WH (1989) Digital image correlation using Newton-Raphson method of partial differential correction. Exp Mech 29:261, doi:10.1007/BF02321405

19. Suo Z, Hutchinson JW (1988) Interface crack between two elastic layers. Int J Fract 43:1-18, doi:10.1007/BF00018123 